12

\title{
Плазменно-иммерсионное формирование высокоинтенсивных ионных пучков
}

\author{
(C) А.И. Рябчиков, П.С. Ананьин, С.В. Дектярев, \\ Д.О. Сивин, А.Э. Шевелев
}

Национальный исследовательский Томский политехнический университет ฯ E-mail: shevelevae@tpu.ru

Поступило в Редакцию 27 декабря 2016 г.

Впервые экспериментально показана возможность формирования высокоинтенсивных пучков ионов металлов и газов низкой энергии. Применение гибридной системы формирования ионного пучка, включающей плазменноиммерсионную экстракцию и ускорение ионов, их последующую баллистическую фокусировку в эквипотенциальном пространстве с обеспечением нейтрализации пространственного заряда пучка, дало возможность импульснопериодического формирования пучков ионов титана и азота с плотностью ионного тока более $1 \mathrm{~A} / \mathrm{cm}^{2}$ и импульсной плотностью мощности $2.6 \mathrm{~kW} / \mathrm{cm}^{2}$.

DOI: 10.21883/PJTF.2017.23.45269.16645

В традиционных методах лучевой ионной имплантации высокоэнергетические ионные пучки формируются путем экстракции ионов из плазмы и их последующего ускорения в высоковольтных системах, состоящих из массива потенциальных сеточных электродов [1-3]. Значительное реальное практическое применение ионной имплантации для модификации свойств металлов и сплавов было достигнуто в результате развития метода плазменно-иммерсионной ионной имплантации [4-6].

В работе [7] на основе теоретического анализа процессов облучения мишеней интенсивными пучками ионов низкой энергии пока- 
зано, что при увеличении плотности тока пучка ионов до $0.6 \mathrm{~A} / \mathrm{cm}^{2}$ при длительности импульса $5 \mu$ s возможно даже изменение механизма массопереноса внедряемой примеси с диффузионного на ударноволновой. В связи с этим разработка метода формирования импульснопериодических высокоинтенсивных пучков ионов газов и металлов низкой энергии представляет значительный интерес как для сверхвысокодозовой имплантации ионов, так и для сверхвысокоскоростного осаждения покрытий.

В настоящей работе описан новый подход к формированию интенсивных пучков ионов металлов и газов с высокой плотностью ионного тока на мишени при использовании газовой плазмы дугового источника с накаленным катодом и металлической вакуумно-дуговой плазмы.

Экспериментальная установка схематично представлена на рис. 1. Для формирования плотной металлической плазмы применялся непрерывный вакуумно-дуговой разряд. Титановый водоохлаждаемый катод располагался по оси испарителя в области магнитного поля напряженностью порядка $60 \mathrm{G}$. Ток дугового разряда составлял 160 А. Металлический сеточный электрод в виде части сферы устанавливался по оси системы (на расстоянии $40 \mathrm{~cm}$ от торцевой поверхности катода генератора плазмы). Плазма азота формировалась с помощью дугового источника с накаленным катодом ПИНК [8] при токе разряда $20 \mathrm{~A}$. В случае газоразрядной плазмы сеточный электрод располагался на расстоянии $9 \mathrm{~cm}$ от выхода генератора плазмы. В экспериментах использован сеточный электрод из нержавеющей стали с радиусом кривизны $7.5 \mathrm{~cm}$ и размером ячейки сетки $1.4 \mathrm{~mm}$.

В отличие от традиционных методов плазменно-иммерсионной имплантации (см., например, [9]) сеточный электрод выступает только в роли экстрактора ионов, а не облучаемой мишени. После прохождения через электрод ионы входят в эквипотенциальное пространство дрейфа и направляются на мишень, установленную вблизи фокуса сеточного электрода. Выпуклая форма сеточного электрода при определенных условиях может обеспечить баллистическую фокусировку ионного пучка. Характерные осциллограммы импульсов тока для пучков ионов титана и азота, полученные с фокусирующим электродом радиусом $7.5 \mathrm{~cm}$ при потенциале смещения $\varphi=-2.6 \mathrm{kV}$, длительностях импульсов $2,4,6$ и $8 \mu \mathrm{s}$, представлены на рис. $2, a$ и $b$. Осциллограммы тока ионов титана демонстрируют существенное влияние длительности импульса смещения на формирование ионного пучка. При длительностях

Письма в ЖТФ, 2017, том 43, вып. 23 


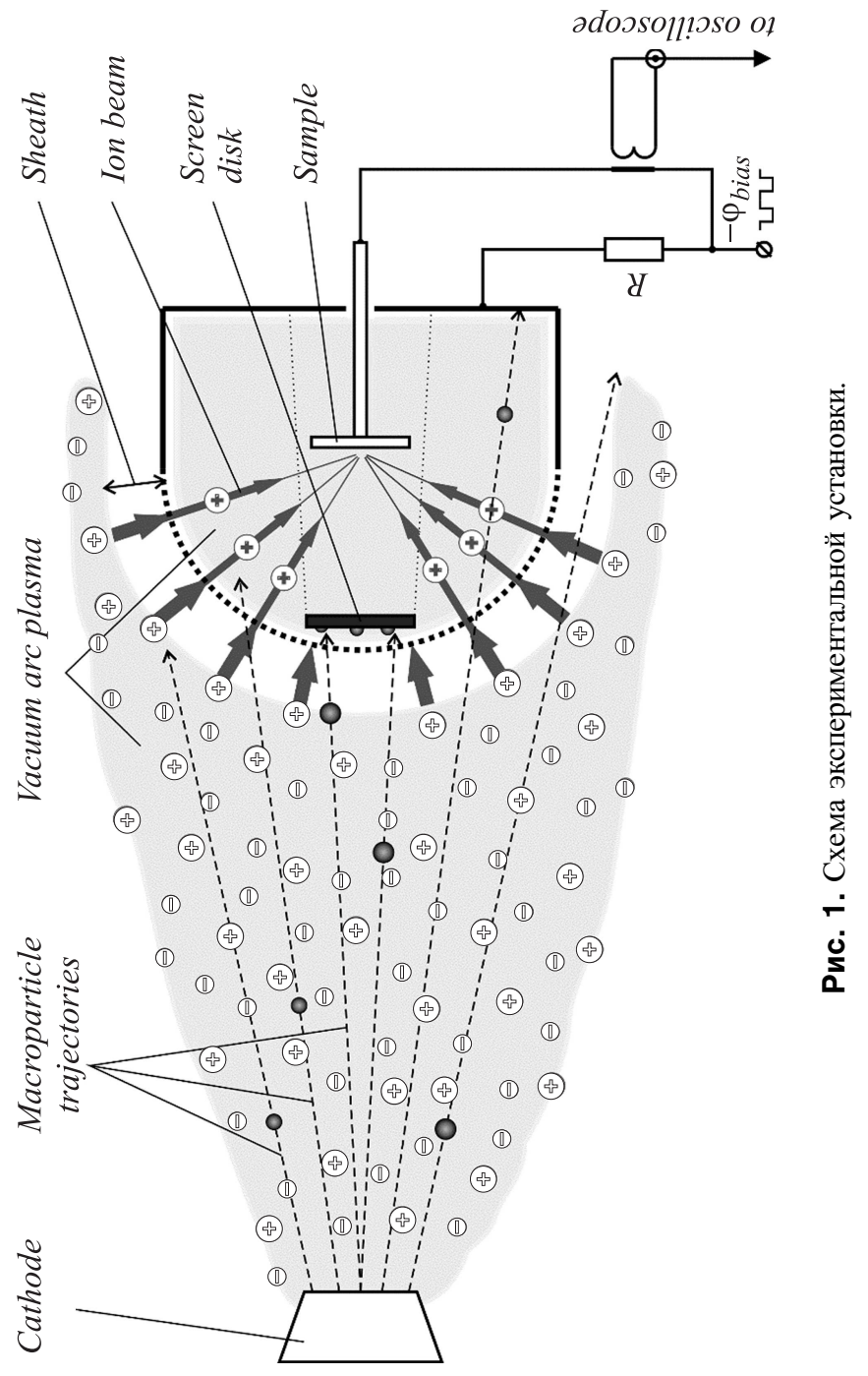

Письма в ЖТФ, 2017, том 43, вып. 23 

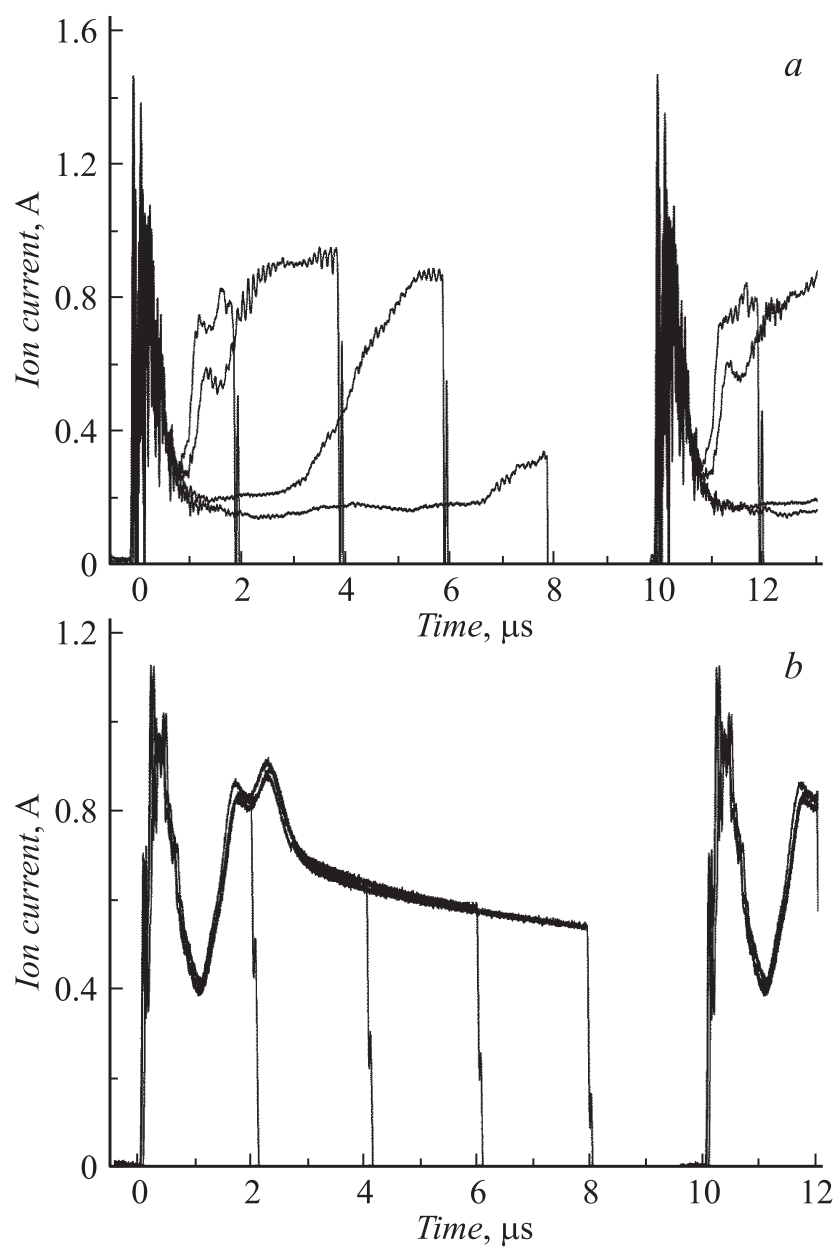

Рис. 2. Осциллограмма импульса тока ионов титана $(a)$ и азота $(b)$ при длительностях ускоряющего импульса $2,4,6,8 \mu \mathrm{s}(-2.6 \mathrm{kV}, 100 \mathrm{kHz})$.

импульсов смещения 2 и $4 \mu$ s формируются ионные пучки с током, достигающим $0.8 \mathrm{~A}$. Увеличение длительности импульса смещения до $6 \mu$ s радикально меняет ситуацию. Амплитуда импульса тока в 
начале импульса снижается в несколько раз. Рост тока начинается почти через $3 \mu \mathrm{s}$. Увеличение длительности импульса смещения до $8 \mu$ s еще больше усугубляет ситуацию. Некоторый рост тока имеет место только в конце $7 \mu \mathrm{s}$.

В случае плазменного потока, формируемого источником ПИНК, наблюдаются высокая стабильность импульсов тока ионов азота и хорошая повторяемость формы импульсов при различных длительностях напряжения смещения. Максимум тока превышает $0.8 \mathrm{~A} \mathrm{в}$ начале импульса, постепенно снижаясь к концу импульса до 0.6 А при длительности импульса $8 \mu \mathrm{s}$. Характерно, что в случае газоразрядной плазмы, когда давление остаточного газа в камере увеличивается до $0.4 \mathrm{~Pa}$, ионный пучок устойчиво формируется и фокусируется даже при длительностях импульсов $8 \mu \mathrm{s}$. Измерения показали, что как в случае ионов титана, так и в случае ионов азота на коллекторе, установленном в фокальной плоскости при амплитуде потенциала смещения $2.6 \mathrm{kV}$, плотность ионного тока может приближаться к $1 \mathrm{~A} / \mathrm{cm}^{2}$. При этом плотность мощности в ионном пучке в импульсе достигает $2.6 \mathrm{~kW} / \mathrm{cm}^{2}$.

Существенное ограничение амплитуды тока ионов титана при длительностях импульса смещения 6 и $8 \mu \mathrm{s}$, по-видимому, связано с изменением условий нейтрализации пространственного заряда ионного пучка. Нейтрализация пространственного заряда является необходимым условием эффективной транспортировки и фокусировки высокоинтенсивного ионного пучка. Отсутствие нейтрализации пространственного заряда пучка с высокой плотностью тока при низком вакууме (случай вакуумной дуги) должно приводить к формированию виртуального анода в области дрейфа пучка на расстояниях, сравнимых с шириной слоя разделения заряда. Инновационность нашего подхода заключается в инжекции плазмы в области дрейфа пучка в паузах между импульсами отрицательного смещения на сеточном электроде. Проникновение плазмы внутрь сеточной структуры обеспечивает наличие холодных плазменных электронов, необходимых для эффективной нейтрализации пространственного заряда пучка и его последующего дрейфа в эквипотенциальном пространстве с баллистической фокусировкой. Поскольку частота импульсов смещения в данных экспериментах была фиксированной, увеличение длительности и импульса потенциала смещения одновременно приводило к уменьшению паузы, в течение которой происходит инжекция плазмы. Учитывая, что скорость непрерывного

Письма в ЖТФ, 2017, том 43, вып. 23 
потока вакуумно-дуговой плазмы обычно порядка $1-1.5 \mathrm{~cm} / \mu \mathrm{s}$, можно оценить, что при длительности импульса смещения $4 \mu$ s плазма в течение паузы $(6 \mu \mathrm{s})$ может пройти расстояние до $9 \mathrm{~cm}$. Это означает, что все пространство между сеточным электродом и коллектором будет заполнено плазмой, обеспечивающей нейтрализацию пространственного заряда ионного пучка. Увеличение длительности импульса смещения до $6 \mu$ s меняет ситуацию. На инжекцию плазмы остается всего $4 \mu \mathrm{s}$. За это время плазма, проникая на расстояние порядка $6 \mathrm{~cm}$, не достигает коллектора. Действие пространственного заряда пучка препятствует его эффективной транспортировке и резко ограничивает ток ионов.

В целом условие, определяющее необходимое время для предварительной инжекции плазмы и заполнения ею всего эквипотенциального пространства в случае непрерывного вакуумного дугового разряда, может быть записано как

$$
\left(\frac{1}{f}-\tau_{\text {bias }}\right) v_{p l} \geqslant R_{e l}
$$

где $f$ - частота следования импульсов, $\tau_{\text {bias }}$ - длительность импульса смещения, $v_{p l}$ - скорость расширения плазмы, $R_{e l}-$ радиус сеточного электрода. В настоящей работе представлены экспериментальные результаты, полученные с использованием непрерывной металлической и газовой плазмы.

В случае газоразрядной плазмы, когда давление остаточного газа в экспериментальной камере повышается из-за напуска газа, на компенсацию пространственного заряда микросекундного фокусируемого ионного пучка дополнительно влияют процессы ионизации газа внутри сеточной структуры. Именно это обстоятельство снимает проблему ограничения тока высокоинтенсивного пучка ионов собственным пространственным зарядом.

Еще один новаторский подход, использованный в данной работе, нацелен на получение высокоинтенсивных пучков ионов металлов, очищенных от макрочастиц вакуумного дугового разряда. В отличие от многообразных низкоэффективных электромагнитных систем сепарации плазмы вакуумной дуги от микрокапельной фракции [10] в настоящей работе впервые применена высокоэффективная система отделения макрочастиц от пучка за счет особенностей формирования

Письма в ЖТФ, 2017, том 43, вып. 23 
ионного потока. Авторы использовали систему типа „солнечного затмения“. Между катодом вакуумного дугового испарителя и коллектороммишенью на внутренней поверхности сеточного электрода по оси системы установлен металлический диск. Радиус диска выбирается из условия исключения возможности прямого попадания макрочастиц с рабочей поверхности катода на мишень в область сфокусированного пучка. Иногда геометрия диска может быть более сложной, если, например, необходимо учесть искривление траектории летящих с катода вакуумного дугового разряда макрочастиц под действием силы тяжести. Полная очистка ионного пучка от макрочастиц вблизи фокальной плоскости пучка была подтверждена данными, полученными при помощи сканирующего электронного микроскопа. Поскольку площадь диска, препятствующего прямому пролету макрочастиц в реальных системах, будет на порядок меньше общей площади сеточного электрода, можно полагать, что эффективность использования плазмы будет высокой и потери пучка не превысят 10\%.

Метод, описанный в работе, предполагает простой и низкозатратный подход к формированию импульсных и импульсно-периодических очищенных от макрочастиц пучков ионов металлов и газов с очень высокой плотностью тока. Экспериментально показана возможность формирования ионных пучков со средней энергией ионов азота $2.6 \mathrm{keV}$ и ионов титана $5.5 \mathrm{keV}$ (с учетом зарядового состояния) с плотностью ионного тока до $1 \mathrm{~A} / \mathrm{cm}^{2}$ и импульсной плотностью мощности до $2.6 \mathrm{~kW} / \mathrm{cm}^{2}$ при средней плотности мощности в ионном пучке более $1 \mathrm{~kW} / \mathrm{cm}^{2}$.

Работа выполнена при финансовой поддержке Министерства образования и науки РФ в рамках государственного задания 3.2415.2017 4.6.

\section{Список литературы}

[1] Poate J.M., Foti G., Jacobson D.C. // Surface modification and alloying by laser, ion, and electron beams / Eds J.H. Poate, G. Foti, D.C. Jacobson. Berlin: Springer, 2013. Book 8. P. 414.

[2] Williams J.S., Poate J.M. // Ion implantation and beam processing / Eds J.S. Williams, J.M. Poate. Academic Press, 2014. P. 419.

[3] Горшков О.Н., Шенина М.Е., Касаткин А.П. и др. // Письма в ЖТФ. 2015. T. 41. B. 11. C 62-70.

[4] Conrad J.R., Radtke J.L., Dodd R.A. et al. // J. Appl. Phys. 1987. V. 62. P. 4591.

Письма в ЖТФ, 2017, том 43, вып. 23 
[5] Арбузов Н.М., Ваулин В.А., Исаев Г.П., Кузьмин О.С., Рябчиков А.И. Патент СССР № 1412517. 1990. Бюл. № 33.

[6] Бурдюх С.В., Стефанович Г.Б., Пергамент А.Л. и др. // Письма в ЖТФ. 2016. T. 42. B. 1. C. 65-71.

[7] Анищик B.М., Углов В.В. // Модификация инструментальных материалов ионными и плазменными пучками. Минск: Изд-во БГУ, 2003. С. 177.

[8] Goncharenko I.I., Grigoriev S.V., Lopatin I.V., Koval N.N. et al. // Surf. Coat. Technol. 2003. V. 169-170. P. 419-423.

[9] Арбузов Н.М., Исаев Г.П., Рябчиков А.И. // Изв. вузов. Физика. 1989. Т. 32. B. 8. C. $68-74$.

[10] Anders A., Anders S., Brown I. // Plasma Sources Sci. Technol. 1995. V. 4. P. $1-12$. 